Cite this: Nanoscale, 2014, 6, 5592

\title{
Quantum dots on vertically aligned gold nanorod monolayer: plasmon enhanced fluorescence
}

Received 29th November 2013

Accepted 7th February 2014

\author{
Bo Peng, ${ }^{a}$ Zhenpeng Li, ${ }^{a}$ Evren Mutlugun, ${ }^{c}$ Pedro Ludwig Hernández Martínez, ${ }^{c}$ \\ Dehui Li, ${ }^{a}$ Qing Zhang, ${ }^{a}$ Yuan Gao, ${ }^{\text {ac }}$ Hilmi Volkan Demir ${ }^{\star a c d}$ and Qihua Xiong ${ }^{\star a b}$
}

DOI: $10.1039 / \mathrm{c} 3 \mathrm{nr} 06341 \mathrm{k}$

www.rsc.org/nanoscale

CTAB-coated Au nanorods were directly self-assembled into a vertically aligned monolayer with highly uniform hot spots through a simple but robust approach. By coupling with $\mathrm{CdSe} / \mathrm{ZnS}$ quantum dots, a maximum enhancement of 10.4 is achieved due to: increased excitation transition rate, radiative rate, and coupling efficiency of emission to the far field.

Plasmonics exhibit the potential to manipulate optics on the sub-wavelength scale, promising exciting applications including negative refractive index media and metamaterials. ${ }^{1-5}$ The self-assembly of monodispersed nanoparticles into ordered structures is an effective approach for designing plasmonics. ${ }^{6-9}$ Spherical Au nanoparticles have been manipulated to generate a diverse selection of plasmonic topologies such as superlattice sheets, ${ }^{10,11}$ chain networks, ${ }^{12}$ and chiral pyramidal structures. ${ }^{13}$ However, ordered assemblies of anisotropic nanostructures, such as Au nanorods, are still very challenging, because the anisotropic shape results in various assembling behaviours. By the Langmuir-Blodgett technique, ${ }^{14,15}$ hydrophobic polymermodified Au nanorods were self-assembled into monolayer sheets consisting of random horizontal and vertical Au nanorods at the air-water interface ${ }^{16}$ Recently, based on the coffeering effect, the evaporation-induced self-assembly has been developed. ${ }^{17}$ During evaporation of the solvent, a flow from the inner region replenishes the liquid that is evaporated at the edge. As a result, nanorods are transferred to the droplet edge and self-assembled into ordered multilayer parallel arrays. ${ }^{18,19}$

${ }^{a}$ Division of Physics and Applied Physics, School of Physical and Mathematical Sciences, Nanyang Technological University, Singapore 637371. E-mail: hvdemir@ ntu.edu.sg; Qihua@ntu.edu.sg

${ }^{b}$ NOVITAS, Nanoelectronics Centre of Excellence, School of Electrical and Electronic Engineering, Nanyang Technological University, Singapore 639798

${ }^{c}$ LUMINOUS, Centre of Excellence for Semiconductor Lighting and Displays, School of Electrical and Electronic Engineering, Nanyang Technological University, Singapore 639798, Singapore

${ }^{d}$ Bilkent University, Department of Physics, Department of Electrical and Electronics Engineering, UNAM - Institute of Materials Science and Nanotechnology, TR-06800, Ankara, Turkey
Meanwhile, a near-equilibrium status is also formed at the internal region of the drying droplet, which contributes to the self-assembly of free anisotropic nanostructures to their lowest energy state in solution. A few previous studies have reported the formation of multilayer vertical arrays by decreasing the electrostatic repulsive force via modifying Au nanorods with weak polar ligands instead of cetyltrimethyl-ammonium bromide (CTAB). ${ }^{20,21}$ Until recently, there have only been a few reports on the directional self-assembly of CTAB-stabilized Au nanorods into vertically aligned multilayer arrays. ${ }^{22,23}$ Recently, our group has shown that it is possible to directly self-assemble CTAB-coated $\mathrm{Au}$ nanorods into highly organized vertical monolayer arrays by synergistically controlling the electrostatic repulsive force and the van der Waals attractive force. ${ }^{24}$

In the past decade, plasmonics has been proven to enhance or quench fluorescence as a function of the distance between the surface plasmons and fluorophores. ${ }^{25-30}$ The previous reports focused their research on the interactions between fluorophores and single Au nanoparticles, ${ }^{31}$ single silver nanoprisms, ${ }^{32}$ and Au disks or hole arrays. ${ }^{33}$ However, a handful of works reported the resonant energy transfer based on the metallic colloid arrays as a surface plasmonic system. ${ }^{34,35}$ Moreover, many colloid substrates suffer from poor reproducibility of "hot spots". Herein, we demonstrate a simple yet robust approach to directionally assemble CTAB-coated Au nanorods into a vertically aligned monolayer and investigate the energy transfer between plasmonic array and monolayer CdSe/ $\mathrm{ZnS}$ quantum dots (QDs). Vertically aligned $\mathrm{Au}$ nanorod monolayers exhibit a strong, reproducible, and highly homogeneous distribution of hot spots. Our experiments show that $\mathrm{Au}$ nanorods with an aspect ratio from 2.1 to 3.2 can be selfassembled into a vertically aligned monolayer and the electrostatic force and van der Waals force predominate in the selfassembly. The $\mathrm{SiO}_{2}$ films were coated on the vertically aligned Au nanorod monolayer by sputter deposition as a spacer to tune the distance between the plasmonic monolayer and QDs. A distinct plasmonic enhancement property was uncovered and a maximum fluorescence enhancement of 10.4 was achieved at a 
$20 \mathrm{~nm} \mathrm{SiO}{ }_{2}$ spacer. Fluorescence-lifetime imaging microscopy (FLIM) was used to measure the lifetime of QDs, which decreased from $\sim 4.1 \mathrm{~ns}$ to minimum, $\sim 0.9 \mathrm{~ns}$, due to the increased radiative decay rate. Therefore, the plasmonic monolayer arrays, due to its enhanced optical density of states, can serve as 2D optical materials such as directionality controller, light enhancers, colour filters, and ultrasensitive SERS sensors. ${ }^{36-38}$

Based on the evaporation-induced strategy, CTAB-coated Au nanorods with an aspect ratio of $\sim 2.7$, which were synthesized by the seeded growth method, ${ }^{39}$ were self-assembled into a vertically aligned monolayer on Si substrates when the Debye length was $3.0 \mathrm{~nm}$ (Fig. 1a). The Au nanorods in the monolayer are aligned to a hexagonal close-packed structure. The inset in Fig. 1a shows a 3-D schematic diagram of the arrays. The interior gap distance between two adjacent $\mathrm{Au}$ nanorods is $\sim 7.7 \mathrm{~nm}$. Fig. 1b exhibits the monolayer structure in a vivid fashion. Surprisingly, the vertically aligned hexagonal $\mathrm{Au}$ nanorod bi-layer was formed in the case when the Debye length decreased to $1.3 \mathrm{~nm}$ by adding $\mathrm{NaCl}$ (Fig. 1c) and the interior gap distance is $\sim 3.4 \mathrm{~nm}$, which corresponds to the length of a CTAB bi-layer. ${ }^{17,40}$ Our approach can be extended to other Au nanorods with different aspect ratios. Fig. 1d shows the absorption spectra of different Au nanorods. The longitudinal plasmon bands are 656, 680, 717, 755 and $780 \mathrm{~nm}$, where the aspect ratios are 2.1, 2.3, 2.7, 2.9 and 3.2, respectively. And the diameters of the corresponding Au nanorods are $45.4,42.6$, $39.8,32.3$ and $27.3 \mathrm{~nm}$, while the lengths are $\sim 94.2,96,100.5$, 94.1 , and $88.2 \mathrm{~nm}$, respectively.

During the self-assembly of $\mathrm{Au}$ nanorods, there are three forces: van der Waals force, depletion force and electrostatic force, whose energy is defined by $E_{\mathrm{vdW}}, E_{\mathrm{dep}}, E_{\text {ele }}$, respectively. The van der Waals and the depletion force are attractive forces and the electrostatic force is a repulsive force. The attractive forces
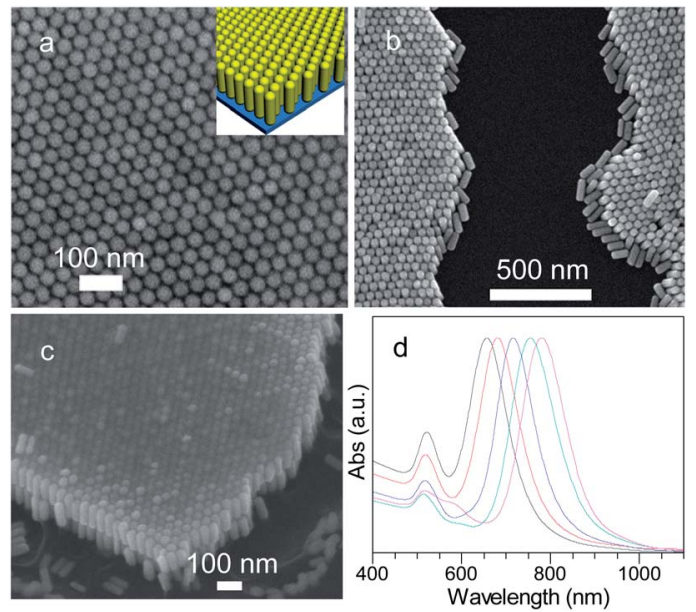

Fig. 1 SEM image of a vertically aligned Au nanorod monolayer: (a) top view, (b) view from the edge. The insets in (a) show the schematic picture of a vertically aligned self-assembled Au nanorod monolayer. (c) The side-view SEM image of vertically aligned hexagonal Au nanorod bi-layer. (d) The absorption spectra of Au nanorod aqueous solution. The longitudinal plasmon bands are 656, 680, 717, 755 and $780 \mathrm{~nm}$, respectively. push Au nanorods to approach each other, whereas the repulsive forces make the nanorods moving away from each other. Therefore, the synergy between the repulsive force and attractive force ensures the alignment of $\mathrm{Au}$ nanorods at the equilibrium status, rather than a random aggregation. We have calculated $E_{\mathrm{vdW}}, E_{\mathrm{dep}}$ and $E_{\text {ele }}$ for five kinds of Au nanorods with 656, 680, 717,755 and $780 \mathrm{~nm}$ plasmon band as a function of edge-to-edge gap size in the case that the Debye length was $3.0 \mathrm{~nm}$. $E_{\mathrm{vdw}}$ and $E_{\text {dep }}$ can be obtained from previous reports. ${ }^{20,41}$ For all Au nanorods, $E_{\mathrm{vdW}}$ is much larger than $E_{\mathrm{dep}}$. Both $E_{\mathrm{vdw}}$ and $E_{\mathrm{dep}}$ increase as the gap size decreases (Fig. 2a). But $E_{\mathrm{vdw}}$ and $E_{\text {dep }}$ decrease as the plasmon band increases from 656 to $780 \mathrm{~nm}$. To calculate $E_{\text {ele }}$ between two adjacent Au nanorods, Derjaguin's approximation is used. $^{42}$ We assume the Au nanorod consists of many slices of parallel thin plates, which contribute to $E_{\text {ele }}{ }^{40}$ Fig. $2 \mathrm{c}$ shows that $E_{\text {ele }}$ decreases with the increasing gap size and also decreases as the plasmon band of Au nanorods increases from 656 to $780 \mathrm{~nm}$. We define the total interaction energy by $E_{\mathrm{total}}=E_{\mathrm{vdW}}+E_{\mathrm{dep}}+E_{\text {ele }}$, which first decreases to a minimum and then increases as the gap size increases. However, for all $\mathrm{Au}$ nanorods with different plasmon bands, the total energy is minimized at $7.5 \mathrm{~nm}$ gap size, which indicates that all Au nanorods can be self-assembled into a vertically aligned monolayer. When the Debye length is decreased to $1.3 \mathrm{~nm}$ by adding $\mathrm{NaCl}$, the calculated minimum $E_{\text {total }}$ is achieved at a gap size of $3.9 \mathrm{~nm}$. However, the lowest limit we can achieve in an aligned $\mathrm{Au}$ nanorod monolayer is $\sim 6.7 \mathrm{~nm}$. Therefore, a vertically aligned $\mathrm{Au}$ nanorod bi-layer is formed to achieve the lowest energy state. In our experiments, the edge-toedge gap size of bi-layer arrays is $\sim 3.4 \mathrm{~nm}$ in the case that the Debye length is $1.3 \mathrm{~nm}$. We suggest that the CTAB molecules are inter-digitated when they approach to touch each other, which leads to a strong depletion force. ${ }^{17}$ Therefore, the experimental gap size is a little smaller than the calculated data.

We focused on the monolayer arrays consisting of $\mathrm{Au}$ nanorods with a longitudinal plasmon band at $717 \mathrm{~nm}$ and
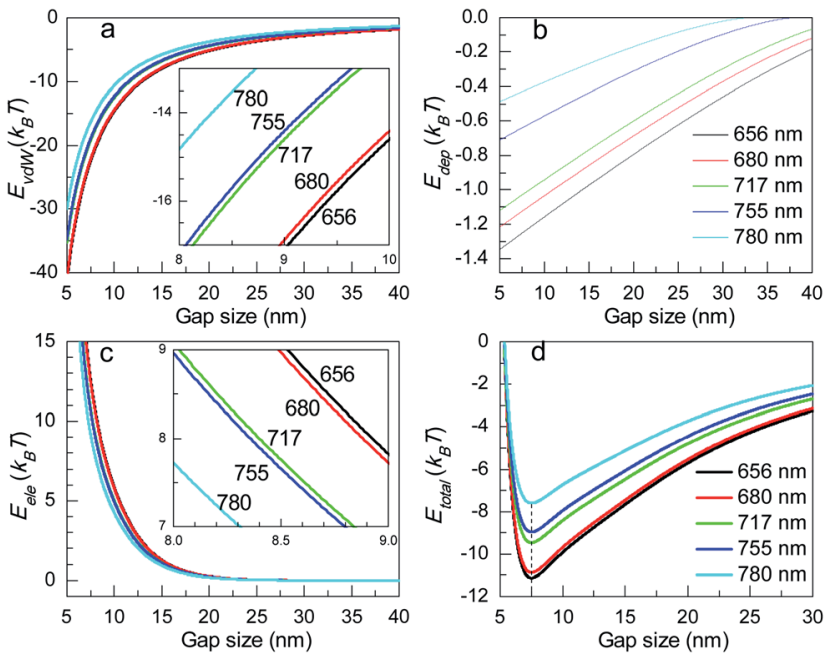

Fig. 2 Interaction free energy as a function of the gap size in the case when the Debye length $k^{-1}$ is $3 \mathrm{~nm}$ : (a) van der Waals force, $E_{\mathrm{vdw}}$, (b) depletion force, $E_{\mathrm{dep}}$, (c) electrostatic repulsive force, $E_{\mathrm{ele}}$ (d) total interaction energy, defined by $E_{\text {Total }}=E_{\mathrm{vaw}}+E_{\text {dep }}+E_{\text {ele }}$. 
investigated the energy transfer between plasmonic arrays and monolayer $\mathrm{CdSe} / \mathrm{ZnS}$ QDs. Sputtered $\mathrm{SiO}_{2}$ layer was used as a spacer to control the distance between the plasmonic monolayer and QDs (Fig. 3a). $10 \mu \mathrm{L} \mathrm{CdSe/ZnS} \mathrm{QDs} \mathrm{hexane} \mathrm{dispersion}$ was drop-cast onto the surface of acetonitrile in a Teflon well $\left(\sim 2 \times 2 \times 2 \mathrm{~cm}^{3}\right) .{ }^{6}$ A monolayer quantum dot (QD) selfassembled film (a typical TEM image is shown in Fig. 3b) was formed within $5 \mathrm{~s}$ and then transferred onto the vertically aligned $\mathrm{Au}$ nanorod monolayer. The absorption peak of the vertically aligned monolayer consisting of $\mathrm{Au}$ nanorod with $717 \mathrm{~nm}$ plasmon band is $\sim 610 \mathrm{~nm}$ checked by CRAIC 20 microspectrophotometer (Fig. 3c). The polarization of white light was parallel to the interparticle distance. Therefore, the transverse plasmon modes interact attractively and these underwent a red shift from $\sim 520$ to $\sim 610 \mathrm{~nm}^{43}$ The photoluminescence (PL) of CdSe/ZnS QD monolayer with and without plasmonic coupling was measured by a home-built system. A $532 \mathrm{~nm}$ laser was used as the excitation, whose polarization direction was along the side of Au nanorod hexagon in the vertically aligned monolayer. Fig. 3d shows room-temperature steady-state PL spectra of CdSe/ZnS QD monolayer on $\mathrm{Si}$ substrates and the vertically aligned Au nanorod monolayer, respectively. The PL peak is at $\sim 614 \mathrm{~nm}$, which is close to the absorption peak of $\mathrm{Au}$ rod arrays. Therefore, a maximum coupling between PL and the scattering of the vertically aligned $\mathrm{Au}$ nanorod monolayer can be achieved, which influences the enhancement of QD emission. ${ }^{44}$ A pronounced $\mathrm{SiO}_{2}$ spacer dependence is spotted from Fig. $3 \mathrm{~d}$. We evaluate the enhancement factor (EF) using $\mathrm{EF}=I / I_{0}$, where $I$ and $I_{0}$ are the PL intensities of CdSe/ZnS QD monolayer with and without the vertically aligned Au nanorod monolayer, respectively. The PL intensities are extracted and $\mathrm{EF}$ is plotted versus $\mathrm{SiO}_{2}$ spacer in Fig. 3e. When the $\mathrm{SiO}_{2}$ spacer thickness is $\sim 5 \mathrm{~nm}$, the PL of the QD monolayer on vertically aligned Au nanorod monolayer is quenched..$^{22}$ When we systematically change the separation between QDs and the plasmonic array, we have clearly observed the distance dependent PL enhancement. The PL intensity with plasmonic coupling at a $10 \mathrm{~nm} \mathrm{SiO}_{2}$ spacer is slightly larger than that of QD monolayer without plasmonic coupling. When the $\mathrm{SiO}_{2}$ spacer is further increased to $\sim 50 \mathrm{~nm}$, the PL intensity of QD monolayer on the vertically aligned Au nanorod monolayer is comparable to the PL signal of the QD monolayer on $\mathrm{Si}$ substrates within the experimental error, which indicates that the plasmonic coupling has barely any effect to the PL when the spacer is larger than $\sim 50 \mathrm{~nm}$. In our experiments, PL exhibits a local maximum at $\sim 20 \mathrm{~nm} \mathrm{SiO}_{2}$ spacer as also in agreement with the previous reports. ${ }^{35,45}$ Generally speaking, the incident light is confined into a small spatial space by the surface plasmon, resulting in a strong local electromagnetic field, $|E|$. The coupling of the excited dipole with plasmons not only has a great effect on both the nonradiative and radiative mode of the transition dipole, but also increases the absorption of incident light. ${ }^{46}$ The competition of the nonradiative and radiative mode determines PL quenching and enhancement. In the case of PL
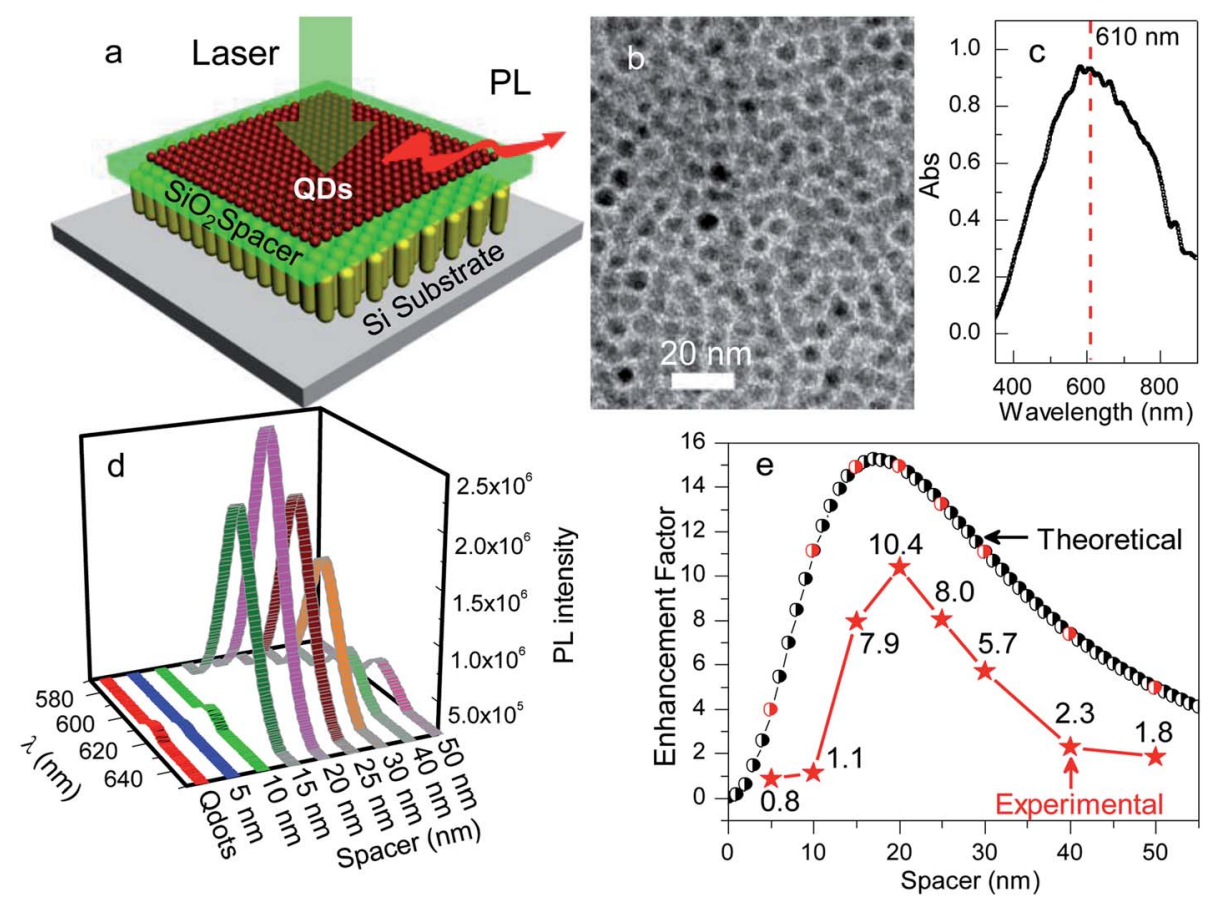

Fig. 3 (a) Schematic picture of the coupling of vertically aligned Au nanorod monolayer and $\mathrm{CdSe} / \mathrm{ZnS} \mathrm{QDs}_{\text {monolayer films. SiO }}$ layer is used as a spacer to control the distance between the plasmonic monolayer and QDs. (b) TEM image of monolayer films of CdSe/ZnS QDs (QDs) with PL emission at $614 \mathrm{~nm}$. (c) Normalized absorption spectrum of a vertically aligned monolayer consisting of Au nanorods with $717 \mathrm{~nm}$ plasmon band. (d) Photoluminescence spectra of CdSe/ZnS QDs monolayer films on vertically aligned Au nanorod monolayer. The silica spacer thickness is 5 , $10,15,20,25,30,40$, and $50 \mathrm{~nm}$, respectively. Non-monotonous behaviour is observed. (e) Plot of calculation (black line + circle, the red circle show the theoretical data at the experimental spacer thickness) and experimental (red line + star) enhancement factor as a function of silica spacer thickness. 
quenching, the nonradiative energy transfer from fluorophores to plasmonic monolayer predominates. Both the small distance and large spectral overlap between the PL spectra and plasmon band lead to a fast nonradiative energy transfer from the excited dipole to the plasmon. In the case of the PL enhancement, first of all, absorption increases due to the increase of $|E|, \gamma_{\text {exc }} \propto|p \cdot E|^{2}$, where $p$ is the transition dipole moment of the fluorophores. ${ }^{25}$ Second, the radiative rate is enhanced significantly due to the Purcell effect. ${ }^{29,44,47,48}$ Because the radiative rate is related to $|E|^{2}, r_{\text {rad }}^{\mathrm{sp}} \propto|E|^{2} r_{\text {rad }}^{0}$, where the radiative decay rates with and without plasmonic coupling are $r_{\text {rad }}^{\mathrm{sp}}$ and $r_{\text {rad }}^{0}{ }^{33}$ Therefore, the enhancement factors are large enough to exhibit the increase of PL intensity..$^{31,35,46}$ In our case, the plasmon resonance frequency $(\sim 610 \mathrm{~nm})$ corresponds to the emission frequency $(\sim 614 \mathrm{~nm})$ of CdSe/ZnS QDs. Therefore, the coupling of QD emission to the far field is increased by the scattering of Au nanorod monolayer arrays, which also induces PL enhancement. ${ }^{44}$

To theoretically estimate the enhancement factor, we consider two main assumptions. First, we calculate the normalized rate of energy dissipation due to a radiative dipole on top of a metallic surface. Second, we estimate the electric field enhancement due to surface plasmon of the metallic nanorod where the cylinder surface plasmon is approximated to a disk. The total effective electric field of one unit in the vertically aligned monolayer is assumed to be 6 times stronger than one disk because the $\mathrm{Au}$ nanorods are aligned in a hexagonal close packed structure. Therefore, the intensity enhancement factor is defined as

$$
\eta\left(\mathbf{r}, \mathbf{r}^{\prime}, \omega_{\text {excitation }}, \omega_{\text {emission }}\right)=A\left(\mathbf{r}, \omega_{\text {excitation }}\right)\left(\frac{P\left(\mathbf{r}^{\prime}, \omega_{\text {emission }}\right)}{P_{0}\left(\mathbf{r}^{\prime}, \omega_{\text {emission }}\right)}\right)^{-1}
$$

where $P / P_{0}$ is the normalized rate of energy dissipation of a radiative dipole defined as ${ }^{49,50}$

$$
\begin{aligned}
& \frac{P}{P_{0}}=1+\frac{1}{2} \operatorname{Re}\left(\int_{0}^{\infty} \frac{s^{3} \mathrm{~d} s}{\sqrt{1-s^{2}}} r^{(\mathrm{p})}(s) \exp \left[2 i k_{1}\left(\sqrt{1-s^{2}}\right) h\right]\right)+ \\
& \frac{1}{2} \operatorname{Re}\left(\int_{0}^{\infty} \frac{s \mathrm{~d} s}{\sqrt{1-s^{2}}}\left[r^{(\mathrm{s})}(s)-\left(1-s^{2}\right) r^{(\mathrm{p})}(s)\right] \exp \left[2 i k_{1}\left(\sqrt{1-s^{2}}\right) h\right]\right)
\end{aligned}
$$

where $r^{(\mathrm{s})}(s)$ and $r^{(\mathrm{p})}(s)$ are the reflection coefficients for $\mathrm{s}$ - and $\mathrm{p}$ polarized waves, respectively, defined as

$$
\begin{gathered}
r^{(s)}(s)=\frac{k_{1} \sqrt{1-s^{2}}-\sqrt{k_{2}^{2}-s^{2} k_{1}^{2}}}{k_{1} \sqrt{1-s^{2}}+\sqrt{k_{2}^{2}-s^{2} k_{1}^{2}}} \\
r^{(\mathrm{p})}(s)=\frac{\varepsilon_{2} k_{1} \sqrt{1-s^{2}}-\varepsilon_{1} \sqrt{k_{2}^{2}-s^{2} k_{1}^{2}}}{\varepsilon_{2} k_{1} \sqrt{1-s^{2}}+\varepsilon_{1} \sqrt{k_{2}^{2}-s^{2} k_{1}^{2}}}
\end{gathered}
$$

where $\varepsilon_{i}$ and $k_{i}$ are the dielectric constant and wave vector of the medium 1 ( $\mathrm{SiO}_{2}$ spacer) and 2 (Au rod). And the electric field enhancement factor due to the presence of the metallic nanostructure is defined as ${ }^{51}$

$$
A(\mathbf{r}, \omega)=\frac{\int_{V_{\mathrm{QD}}}\left|E_{\text {metal }}(\mathbf{r}, \omega)\right|^{2} \mathrm{~d} V}{\int_{V_{\mathrm{QD}}}\left|E_{0}(\mathbf{r}, \omega)\right|^{2} \mathrm{~d} V}
$$

where $E_{0}(\mathbf{r}, \omega)$ and $E_{\text {metal }}(\mathbf{r}, \omega)$ are the electric field without and with metallic nanostructure, respectively. The electric field for the cylinder with radius " $a$ " is

$$
E(\mathbf{r})=E_{0}\left\{\begin{array}{l}
\frac{1}{1+\left(\frac{z}{a}\right)^{2}} z \quad \rho=0 ; \quad z>0 \\
\int_{0}^{1}\left(\frac{1}{\sqrt{1-\rho_{0}^{2}}}\right)\left(\frac{\left(\frac{\rho}{a}-\rho_{0}\right) \hat{\rho}+\frac{z}{a} \hat{z}}{\left[\left(\frac{\rho}{a}-\rho_{0}\right)^{2}+\left(\frac{z}{a}\right)^{2}\right]^{3 / 2}}\right) \rho_{0} \mathrm{~d} \rho_{0} \\
\rho>0 ; \quad z>0
\end{array}\right.
$$

where $\rho$ is the radial component of the electric field perpendicular to the cylinder axis. $\boldsymbol{Z}$ is the $z$ component of the electric field parallel to the cylinder axis. As shown in Fig. 3e, the numerical calculation results are in good agreement with our experimental data.

To understand the coupling of plasmonic monolayer and QDs, fluorescence-lifetime imaging microscopy was used to obtain time-resolved fluorescence spectroscopy, which is critically dependent on the distance between the plasmonic monolayer and QDs. Fig. 4a shows a microscopic image of the vertically aligned Au nanorod monolayer (yellow color) coated by CdSe/ZnS QD monolayer film. The $\mathrm{SiO}_{2}$ spacer is $10 \mathrm{~nm}$. From the crack band of QD monolayer films, it is clearly shown that the QD monolayer films are darker than Si substrates and cover the vertically aligned $\mathrm{Au}$ nanorod monolayer. Fig. 4b shows a FLIM image of QD monolayer corresponding to Fig. 4a. The crack band of QD monolayer film is vivid. The lifetime of CdSe/ZnS QD monolayer on a vertically aligned Au nanorod monolayer is much shorter than that on Si substrates, which indicates that the lifetime of QDs decreases due to plasmonic interaction. Fig. 4c shows time-resolved PL spectra of CdSe/ZnS QDs. The $\mathrm{SiO}_{2}$ spacer thicknesses are 5, 10, 15, 20, 25, 30, 40 and $50 \mathrm{~nm}$, respectively. All the time-resolved PL decay curve can be well fitted as a bi-exponential function, $I(t)=A_{1} \exp \left(-t / \tau_{1}\right)+$ $A_{2} \exp \left(-t / \tau_{2}\right)$, with two time constants: a fast decay $\left(\tau_{1}\right)$ accompanied by long-lasting emission $\left(\tau_{2}\right)$, where $I(t)$ is PL intensity. ${ }^{52,53}$ The results are consistent with previous studies. The fast decay $\tau_{1}$ is associated with recombination of carriers from the delocalized states in the core region, where the slow decay $\tau_{2}$ originates from recombination of carriers from the localized states at the hetero-interface. ${ }^{54,55}$ The average lifetime is defined by $\tau=\tau_{1} \times a_{1}+\tau_{2} \times a_{2}$, where $a_{1}$ and $a_{2}$ are the fraction of $\tau_{1}$ and $\tau_{2}$, respectively. For CdSe/ZnS QDs on Si substrates, the lifetime is $\tau=4.1 \pm 0.1 \mathrm{~ns}\left(\tau_{1}=2.3 \pm 0.3 \mathrm{~ns}\right.$ and $\tau_{2}=10.3 \pm 2.1$ ns). Fig. 4d shows the lifetime $\tau$ as a function of $\mathrm{SiO}_{2}$ spacer thickness. The lifetime was obtained by measurement and statistical analysis of many CdSe/ZnS QD monolayers on the vertically aligned $\mathrm{Au}$ nanorod monolayer $(>10)$. The decay lifetimes $\tau$ are $2.4 \pm 0.14,2.0 \pm 0.2,1.8 \pm 0.15,0.9 \pm 0.1,1.9 \pm 0.25$, $2.6 \pm 0.16,3.5 \pm 0.21$, and $3.7 \pm 0.2 \mathrm{~ns}$ in the case that $\mathrm{SiO}_{2}$ spacer thickness is $5,10,15,20,25,30,40$ and $50 \mathrm{~nm}$, respectively. The lifetime first achieves a minimum at a $20 \mathrm{~nm}$ spacer and then increases, which corresponds to the PL enhancement 

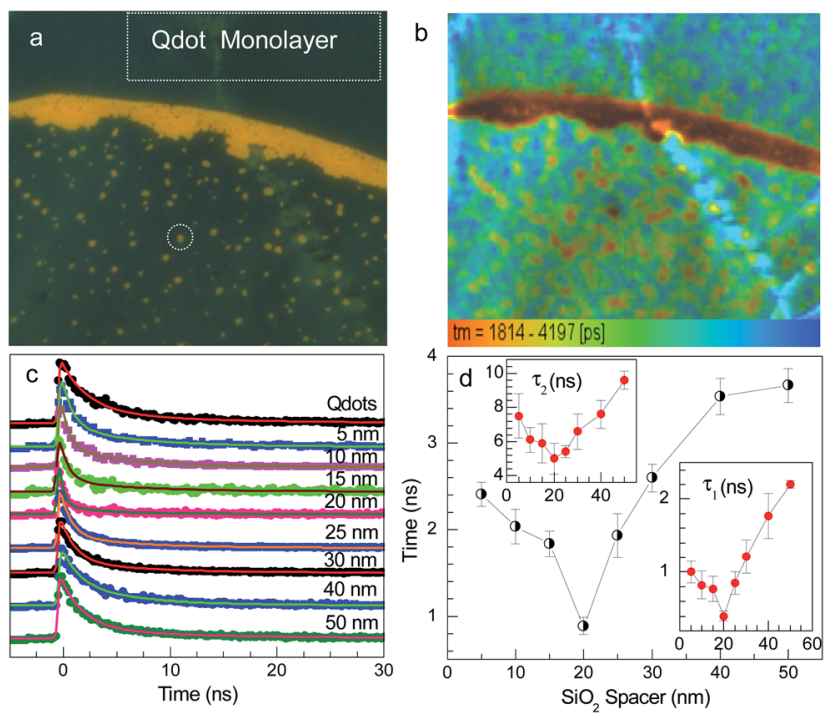

Fig. 4 (a) Optical microscopy image of vertically aligned Au nanorod monolayer covered by $\mathrm{CdSe} / \mathrm{ZnS}$ QD monolayer films. The $\mathrm{SiO}_{2}$ spacer is $10 \mathrm{~nm}$. (b) Fluorescence-lifetime microscopy image of CdSe/ ZnS QD monolayer films corresponding to (a). The corresponding vertically aligned Au nanorod monolayer covered by QD monolayer in (a) are shown in the white dotted circles. The bare CdSe/ZnS QD monolayer is outside of the golden yellow in (a). The lifetime of CdSe/ ZnS QDs is shortened vividly on the vertically aligned Au nanorod monolayer. (c) Time-resolved fluorescence spectra of CdSe/ZnS QD monolayer films on vertically aligned Au nanorod monolayer. The silica spacer thickness is $5,10,15,20,25,30,40$, and $50 \mathrm{~nm}$, respectively. The solid lines are fits to the data using an exponential decay function as described in the text. (d) The average lifetime $\tau$ as a numerical function of the silica spacer thickness. The lifetime was obtained by measurement and statistical analysis of (b). The insets show $\tau_{1}$ and $\tau_{2}$ versus the silica spacer thickness, respectively.

results, where the enhancement of 10.4 times is maximized at a $20 \mathrm{~nm}$ spacer. It is important to note that the trends of $\tau_{1}$ and $\tau_{2}$ as a function of the silica spacer thickness are the same with $\tau$, as shown in the insets in Fig. $4 \mathrm{~d}$. Both $\tau_{1}$ and $\tau_{2}$ are minimized in the case of a $20 \mathrm{~nm}$ silica spacer. The decrease of lifetime corresponds to the pronounced enhancement of PL. Therefore, the increased radiative rate is large enough and contributes to the PL enhancement, although plasmon coupling enhances both the radiative and nonradiative rate ${ }^{32,46}$ Generally speaking, three factors determine the overall PL enhancement: increased transition rate of electrons, increased radiative rate due to the coupling of excitons with plasmons, and enhanced coupling efficiency of the fluorescence emission to the far field. ${ }^{44}$ When CdSe/ZnS QDs are excited by incident photons, the electrons in the valence band transfer into the conduction band. The transition rate can be enhanced by surface plasmons, because the photon absorption of QDs is increased by plasmonic coupling. ${ }^{25}$ Meanwhile, the vertically aligned $\mathrm{Au}$ nanorod monolayer reflects the incident photons to excite CdSe/ZnS QDs again, which also contributes to enhancement of the transition rate. ${ }^{34}$ When exciton recombination induces PL emission, a new modified radiative decay rate is induced due to plasmonic coupling, $r_{\text {rad }}^{\text {new }}$. Therefore, the radiative decay rate is accelerated, $r_{\mathrm{rad}}^{\mathrm{sp}}=r_{\mathrm{rad}}^{0}+r_{\mathrm{rad}}^{\mathrm{new}}$, where $r_{\mathrm{rad}}^{\mathrm{sp}}$ and $r_{\mathrm{rad}}^{0}$ are the radiative decay rate with and without plasmonic coupling, respectively. ${ }^{34}$ Furthermore, the correspondence between plasmon resonance frequency $(\sim 610 \mathrm{~nm})$ and emission frequency $(\sim 614 \mathrm{~nm})$ enhances the coupling efficiency of emission to the far field. Therefore, the PL of CdSe/ZnS QDs is enhanced by plasmons. Therefore, we suggest that the increased excitation transition rate, radiative rate of excition recombination for emission, and coupling efficiency of emission to the far field lead to enhancement of CdSe/ZnS QD monolayer films, which has been proved by previous reports in theory and experiment. ${ }^{33,44,56,57}$

\section{Conclusions}

In summary, vertically aligned $\mathrm{Au}$ nanorod monolayers with $\sim 7.7 \mathrm{~nm}$ edge-to-edge gap size were prepared by an evaporationinduced self-assembly strategy in the internal region of a drying droplet, where a near-equilibrium state is achieved. This approach is extended to Au nanorods with an aspect ratio of 2.1, 2.3, 2.7, 2.9 and 3.2, where the longitudinal plasmon bands are $656,680,717,755$ and $780 \mathrm{~nm}$, respectively. A vertically aligned Au nanorod bi-layer is formed by decreasing the Debye length to $1.3 \mathrm{~nm}$. During the self-assembly of Au nanorods, the electrostatic force and van der Waals force predominate, determining the self-assembling behavior of $\mathrm{Au}$ nanorods. Based on the vertically aligned monolayer consisting of Au nanorods with a longitudinal plasmon band at $717 \mathrm{~nm}$, we investigate the energy transfer between the plasmonic monolayer and CdSe/ZnS QDs. The $\mathrm{SiO}_{2}$ films deposited by sputtering are used as the spacer to control the distance between the plasmonic array and QDs. The $\mathrm{CdSe} / \mathrm{ZnS}$ QD monolayer is coated on the vertically aligned $\mathrm{Au}$ nanorod monolayer. Maximum enhancement of 10.4 times is achieved at the $20 \mathrm{~nm}$ spacer, where the lifetime of CdSe/ZnS QDs is minimized to $0.9 \mathrm{~ns}$. The PL enhancement is due to three components: increased excitation transition rate of electrons from the valence band to the conduction band, increased radiative rate in the case of exciton recombination for emission, and increased coupling efficiency of PL to the far field through scattering of the vertically aligned Au nanorod monolayer.

\section{Acknowledgements}

Q.X. and H.V.D. gratefully acknowledge financial support from the Singapore National Research Foundation via a Competitive Research Program (NRF-CRP-6-2010-2). Q.X. also thanks for support from the Singapore National Research Foundation through a fellowship grant (NRF-RF-2009-06), and the Singapore Ministry of Education via two Tier 2 grants (MOE2011-T2-2051 and MOE2011-T2-2-085). Additionally, H.V.D thankfully acknowledges the Singapore National Research Foundation Fellowship Program (NRF-RF-2009-09).

\section{Notes and references}

1 H. Wang, D. W. Brandl, P. Nordlander and N. J. Halas, Acc. Chem. Res., 2007, 40, 53-62. 
2 M. L. Brongersma and V. M. Shalaev, Science, 2010, 328, 440441.

3 B. Luk'yanchuk, N. I. Zheludev, S. A. Maier, N. J. Halas, P. Nordlander, H. Giessen and C. T. Chong, Nat. Mater., 2010, 9, 707-715.

4 Q. Zhang, X. Wen, G. Li, Q. Ruan, J. Wang and Q. H. Xiong, ACS Nano, 2013, 7, 11071-11078.

5 X. L. Xu, B. Peng, D. H. Li, J. Zhang, L. M. Wong, Q. Zhang, S. J. Wang and Q. H. Xiong, Nano Lett., 2011, 11, 3232-3238.

6 A. Dong, J. Chen, S. J. Oh, W.-K. Koh, F. Xiu, X. Ye, D.-K. Ko, K. L. Wang, C. R. Kagan and C. B. Murray, Nano Lett., 2011, 11, 841-846.

7 A. Tao, P. Sinsermsuksakul and P. Yang, Nat. Nanotechnol., 2007, 2, 435-440.

8 B. Peng, D. Chen, Z. T. Deng, T. Wen, X. W. Meng, X. L. Ren, J. Ren and F. Q. Tang, ChemPhysChem, 2010, 11, 3744-3751.

9 B. Peng, Z. T. Deng, F. Q. Tang, D. Chen, X. L. Ren and J. Ren, Cryst. Growth Des., 2009, 9, 4745-4751.

10 W. Cheng, M. J. Campolongo, J. J. Cha, S. J. Tan, C. C. Umbach, D. A. Muller and D. Luo, Nat. Mater., 2009, 8, 519-525.

11 M. N. Martin, J. I. Basham, P. Chando and S.-K. Eah, Langmuir, 2010, 26, 7410-7417.

12 J. X. Huang, A. R. Tao, S. Connor, R. R. He and P. D. Yang, Nano Lett., 2006, 6, 524-529.

13 S. J. Tan, M. J. Campolongo, D. Luo and W. L. Cheng, Nat. Nanotechnol., 2011, 6, 268-276.

14 P. Yang, Nature, 2003, 425, 243-244.

15 F. Kim, S. Kwan, J. Akana and P. Yang, J. Am. Chem. Soc., 2001, 123, 4360-4361.

16 K. C. Ng, I. B. Udagedara, I. D. Rukhlenko, Y. Chen, Y. Tang, M. Premaratne and W. Cheng, ACS Nano, 2012, 6, 925-934.

17 T. K. Sau and C. J. Murphy, Langmuir, 2005, 21, 2923-2929.

18 B. Nikoobakht, Z. L. Wang and M. A. El-Sayed, J. Phys. Chem. $B, 2000,104,8635-8640$.

19 T. Ming, X. Kou, H. Chen, T. Wang, H.-L. Tam, K.-W. Cheah, J.-Y. Chen and J. Wang, Angew. Chem., Int. Ed., 2008, 47, 9685-9690.

20 Y. Xie, S. Guo, Y. Ji, C. Guo, X. Liu, Z. Chen, X. Wu and Q. Liu, Langmuir, 2011, 27, 11394-11400.

21 A. Guerrero-Martínez, J. Pérez-Juste, E. Carbó-Argibay, G. Tardajos and L. M. Liz-Marzán, Angew. Chem., Int. Ed., 2009, 48, 9484-9488.

22 R. A. Alvarez-Puebla, A. Agarwal, P. Manna, B. P. Khanal, P. Aldeanueva-Potel, E. Carbo-Argibay, N. Pazos-Perez, L. Vigderman, E. R. Zubarev, N. A. Kotov and L. M. LizMarzan, Proc. Natl. Acad. Sci. U. S. A., 2011, 108, 8157-8161.

23 T. Thai, Y. H. Zheng, S. H. Ng, S. Mudie, M. Altissimo and U. Bach, Angew. Chem., Int. Ed., 2012, 51, 8732-8735.

24 B. Peng, G. Li, D. Li, S. Dodson, Q. Zhang, J. Zhang, Y. H. Lee, H. V. Demir, X. Yi Ling and Q. H. Xiong, ACS Nano, 2013, 7, 5993-6000.

25 L. Novotny, P. Anger and P. Bharadwaj, Phys. Rev. Lett., 2006, 96, 113002.

26 B. Peng, Q. Zhang, X. F. Liu, Y. Ji, H. V. Demir, C. H. A. Huan, T. C. Sum and Q. H. Xiong, ACS Nano, 2012, 6, 6250-6259.
27 T. Pons, I. L. Medintz, K. E. Sapsford, S. Higashiya, A. F. Grimes, D. S. English and H. Mattoussi, Nano Lett., 2007, 7, 3157-3164.

28 G. Schneider, G. Decher, N. Nerambourg, R. Praho, M. H. V. Werts and M. Blanchard-Desce, Nano Lett., 2006, 6, 530-536.

29 K. Munechika, Y. C. Chen, A. F. Tillack, A. P. Kulkarni, I. JenLa Plante, A. M. Munro and D. S. Ginger, Nano Lett., 2011, 11, 2725-2730.

30 Q. Zhang, X. Y. Shan, X. Feng, C. X. Wang, Q. Q. Wang, J. F. Jia and Q. K. Xue, Nano Lett., 2011, 11, 4270-4274.

31 S. Kühn, U. Håkanson, L. Rogobete and V. Sandoghdar, Phys. Rev. Lett., 2006, 97, 017402.

32 K. Munechika, Y. Chen, A. F. Tillack, A. P. Kulkarni, I. J. L. Plante, A. M. Munro and D. S. Ginger, Nano Lett., 2010, 10, 2598-2603.

33 Y. K. Wang, T. Y. Yang, M. T. Tuominen and M. Achermann, Phys. Rev. Lett., 2009, 102, 163001.

34 P. F. Guo, S. Wu, Q. J. Ren, J. Lu, Z. H. Chen, S. J. Xiao and Y. Y. Zhu, J. Phys. Chem. Lett., 2010, 1, 315-318.

35 O. Kulakovich, N. Strekal, A. Yaroshevich, S. Maskevich, S. Gaponenko, I. Nabiev, U. Woggon and M. Artemyev, Nano Lett., 2002, 2, 1449-1452.

36 X. Wen, G. Li, J. Zhang, Q. Zhang, B. Peng, L. M. Wong, S. Wang and Q. H. Xiong, Nanoscale, 2014, 6, 132-139.

37 C. Cao, J. Zhang, X. Wen, S. L. Dodson, N. T. Dao, L. M. Wong, S. Wang, S. Li, A. T. Phan and Q. H. Xiong, ACS Nano, 2013, 7, 7583-7591.

38 J. X. Fang, S. Y. Du, S. Lebedkin, Z. Y. Li, R. Kruk, M. Kappes and H. Hahn, Nano Lett., 2010, 10, 5006-5013.

39 T. Ming, L. Zhao, Z. Yang, H. Chen, L. Sun, J. Wang and C. Yan, Nano Lett., 2009, 9, 3896-3903.

40 K. L. Young, M. R. Jones, J. Zhang, R. J. Macfarlane, R. Esquivel-Sirvent, R. J. Nap, J. S. Wu, G. C. Schatz, B. Lee and C. A. Mirkin, Proc. Natl. Acad. Sci. U. S. A., 2012, 109, 2240-2245.

41 K. J. M. Bishop, C. E. Wilmer, S. Soh and B. A. Grzybowski, Small, 2009, 5, 1600-1630.

42 H. Ohshima and A. Hyono, J. Colloid Interface Sci., 2009, 333, 202-208.

43 A. M. Funston, C. Novo, T. J. Davis and P. Mulvaney, Nano Lett., 2009, 9, 1651-1658.

44 F. Tam, G. P. Goodrich, B. R. Johnson and N. J. Halas, Nano Lett., 2007, 7, 496-501.

45 J. R. Lakowicz, K. Ray and R. Badugu, Langmuir, 2006, 22, 8374-8378.

46 K. T. Shimizu, W. K. Woo, B. R. Fisher, H. J. Eisler and M. G. Bawendi, Phys. Rev. Lett., 2002, 89, 117401.

47 R.-M. Ma, R. F. Oulton, V. J. Sorger, G. Bartal and X. Zhang, Nat. Mater., 2011, 10, 110-113.

48 R. Carminati, J. J. Greffet, C. Henkel and J. M. Vigoureux, Opt. Commun., 2006, 261, 368-375.

49 L. Novotny, J. Opt. Soc. Am. A, 1997, 14, 91-104.

50 L. Novotny and B. Hecht, Dipole Emission Near Planar Interfaces, in Principles of Nano-Optics, Cambridge University Press, 2007, pp. 335-346. 
51 A. O. Govorov, G. W. Bryant, W. Zhang, T. Skeini, J. Lee, N. A. Kotov, J. M. Slocik and R. R. Naik, Nano Lett., 2006, 6, 984-994.

52 X. L. Xu, Y. Y. Zhao, E. J. Sie, Y. H. Lu, B. Liu, S. A. Ekahana, X. Ju, Q. K. Jiang, J. B. Wang, H. D. Sun, T. C. Sum, C. H. A. Huan, Y. P. Feng and Q. H. Xiong, ACS Nano, 2011, 5, 3660-3669.

53 A. F. van Driel, I. S. Nikolaev, P. Vergeer, P. Lodahl, D. Vanmaekelbergh and W. L. Vos, Phys. Rev. B: Condens. Matter Mater. Phys., 2007, 75, 035329.
54 G. W. Shu, W. Z. Lee, I. J. Shu, J. L. Shen, J. C. A. Lin, W. H. Chang, R. C. Ruaan and W. C. Chou, IEEE Trans. Nanotechnol., 2005, 4, 632-636.

55 W. Z. Lee, G. W. Shu, J. S. Wang, J. L. Shen, C. A. Lin, W. H. Chang, R. C. Ruaan, W. C. Chou, C. H. Lu and Y. C. Lee, Nanotechnology, 2005, 16, 1517-1521.

56 O. G. Tovmachenko, C. Graf, D. J. van den Heuvel, A. van Blaaderen and H. C. Gerritsen, Adv. Mater., 2006, 18, 91-95.

57 Y. H. Chan, J. X. Chen, S. E. Wark, S. L. Skiles, D. H. Son and J. D. Batteas, ACS Nano, 2009, 3, 1735-1744. 